\title{
Prioritising Low Cost Digital Solutions Required by Manufacturing SMEs: A Shoestring Approach
}

\author{
Benjamin Schönfuß ${ }^{1}$, Duncan McFarlane ${ }^{1}$, Nicky Athanassopoulou ${ }^{1}$, Liz Salter ${ }^{1}$, \\ Lavindra de Silva ${ }^{1}$, and Svetan Ratchev ${ }^{2}$
}

\begin{abstract}
This paper establishes a reference set of those low cost digital solutions needed by small and medium sized manufacturers - SMEs - and proposes a method for determining development priorities using input from reference groups of SMEs. The paper describes the approach taken to identifying and classifying common digital solutions used in manufacturing and the results from a series of workshops in which company representatives prioritise different solution types to help guide developments.
\end{abstract}

Key words: Digital Manufacturing, Low-Cost Automation, Digitalisation Strategy, Manufacturing SMEs

\section{Introduction}

\subsection{Aim}

This paper proposes a method to develop a reference set of low cost digital manufacturing solutions. Our goal is to establish a complete set of digital manufacturing activities an SME could engage in. The solutions are listed and classified in a catalogue. This catalogue helps companies that have identified the need for more digital manufacturing by being a reference to choose their next steps. It also assists companies that still operate in a more traditional way to better understand their options. Finally, this catalogue can help researchers, who wish to do research relevant to industry, to focus on certain aspects of digital manufacturing for their research projects. The catalogue is based on our initial findings regarding the priorities of a large group of SMEs.

\footnotetext{
${ }^{1}$ University of Cambridge $\cdot{ }^{2}$ University of Nottingham
} 


\subsection{Low Cost Digital Solutions for Manufacturing SMEs}

Small and medium sized enterprises are often regarded as the backbone of the UK economy. $99 \%$ of the 5.6 million businesses in the UK are SMEs [15]. However, only a relatively small number of SMEs explore the implementation of advanced digital technologies in their manufacturing process [3,11]. A lack of skilled personnel and the high perceived costs of digitalisation projects are often cited as major reasons [16].

Digital Manufacturing on a Shoestring [5] is an Engineering and Physical Sciences Research Council (EPSRC) funded research project that seeks to help SMEs by making digital manufacturing more accessible [6]. The focus of the investigation is the application of low cost, easily accessible, "off-the-shelf" components in the manufacturing environment. Figure 1 shows the approach behind the project. We first analyse which digital manufacturing solutions are of high priority to SMEs. We then identify technology building blocks that are needed for these solutions, focussing on keeping the cost low. The final output is a set of methods that define how the building blocks can be combined flexibly to implement the solutions within a business.

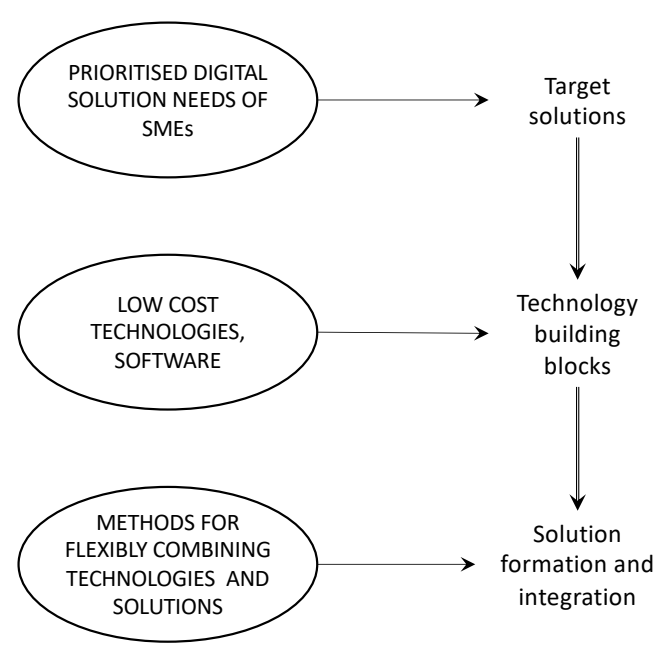

Fig. 1 The Shoestring approach [13]

Prioritising the solutions required by SMEs is the first work stream of the Digital Manufacturing on a Shoestring project. The objective is to ensure the relevance of further technical developments by identifying digital manufacturing solutions that are suitable and of high priority for manufacturing SMEs. The second objective is to help SMEs select specific steps that they should pursue if they wish to implement more digital technologies. 


\subsection{Outline of the Paper}

In the next section, we give an overview of SME-specific challenges in digital manufacturing. We then propose a method to develop and rank a catalogue of potential digital manufacturing solutions. We show existing approaches to digital manufacturing and automation assessment.

We then introduce the initial classification approach taken to group the catalogue of digital manufacturing solutions into relevant parts for each individual application. We present the results of the first data capture activities with SMEs and the implications for future research in digital manufacturing and automation.

Finally, current limitations and an outlook of the next steps in the project are presented.

For the purpose of this paper, Digital Manufacturing and Digitalisation are defined as the shift of business processes and operations to exploit the potential of digital technologies.

\section{Digital Manufacturing Requirements of SMEs}

SMEs are typically differentiated from larger companies by their headcount, annual turnover and assets on the balance sheet. The European Union for example defines SME as shown in table 1 . This is also the definition adopted in this paper.

Table 1 Definition of SMEs depending on size criteria according to the European Commission [14]

\begin{tabular}{|l|l|l|l|}
\hline Company Size & Headcount & Annual Turnover & Annual Balance Sheet Total \\
\hline Medium-Sized & $<250$ & $\leq € 50 \mathrm{MN}$ & $\leq € 43 \mathrm{MN}$ \\
Small & $<50$ & $\leq € 10 \mathrm{MN}$ & $\leq € 10 \mathrm{MN}$ \\
Micro & $<10$ & $\leq € 2 \mathrm{MN}$ & $\leq € 2 \mathrm{MN}$ \\
\hline
\end{tabular}

This size difference leads to special challenges that SMEs face when considering the implementation of digital manufacturing technologies into their processes. Their typically lower resources and access to capital is a barrier for SMEs to make investments into high tech manufacturing technologies $[1,10,12]$. Their lower bargaining power can make investments prohibitively costly [4]. SMEs often lack the internal skills to plan and implement new technologies [9]. The typical product mix of many SMEs (low volume, high complexity) and their often lacking process standardisation impede automation projects further $[2,10]$.

Despite these challenges, we observed in preliminary workshops, interviews and visits to SMEs that the interest in digital manufacturing is very high and potential productivity gains are expected by many company owners. Even with the high diversity of SMEs, many of them seem to share similar problems that can be ad- 
dressed by digital solutions. This opens the opportunity for standardised, low cost developments to address these problems.

\section{Methodology for Classification and Prioritisation of Digital Manufacturing Solutions}

\subsection{Overview}

There are many existing approaches, frameworks and tools with the objective to help companies decide what they should do in the area of digital manufacturing. To determine if any existing tool is suitable to guide the research in this project, we have conducted a screening based on the factors shown in table 2 . The tools were suggested by users, consultants and researchers from the UK as well as sourced from industry and government reports on digital manufacturing. A total of 18 tools were evaluated.

Table 2 Factors to determine the fitness of existing digital assessment tools for the Digital Manufacturing on a Shoestring project

\begin{tabular}{|l|l|}
\hline Factor & Guiding question \\
\hline \hline Target company & What type of company is the tool geared for? (size / sector) \\
\hline Unit of analysis & Which part of the company is analysed? (shop floor / whole company / ...) \\
\hline Focus of the tool & What exactly is the objective of the tool? \\
\hline Specificity of output & What is the type of output? (board overview / specific action steps / ...) \\
\hline Flexibility of delivery & Can longer and shorter versions be derived to increase engagement? \\
\hline License & Is the tool in the public domain? \\
\hline Time commitment & How much time is needed from the participants' point of view? \\
\hline Deliverables & What is the tangible output? (report / score / ...) \\
\hline Form of delivery & How do participants interact with the tool? (workshop / online / ...) \\
\hline Assessment approach & What is the basis for assessment? (scoring system / model / ...) \\
\hline User of the tool & Who can use the tool? (trained consultants / practitioners / ...) \\
\hline Development status & Is the tool ready to be used? \\
\hline Number of uses & How often was the tool used so far? \\
\hline Limitations & What are known limitations of the tool? \\
\hline
\end{tabular}

No tool individually provided the level of detail needed to provide SMEs with specific action steps in combination with the ease of use that is necessary to get companies to engage. We therefore looked for individual exercises and activities from the evaluated tools that can be combined into a research method for our project. The focus was on activities that can be used to assess the whole company, that can 
be delivered flexibly and that are under a suitable licensing agreement that allows use for research activities and publishing of the results.

Next, we describe the method we used for developing a prioritised set of digital manufacturing solutions for SMEs. The method is designed to be scalable to allow engagement with as many SMEs as possible. This is important to get a better estimation of the needs of the whole SME population.

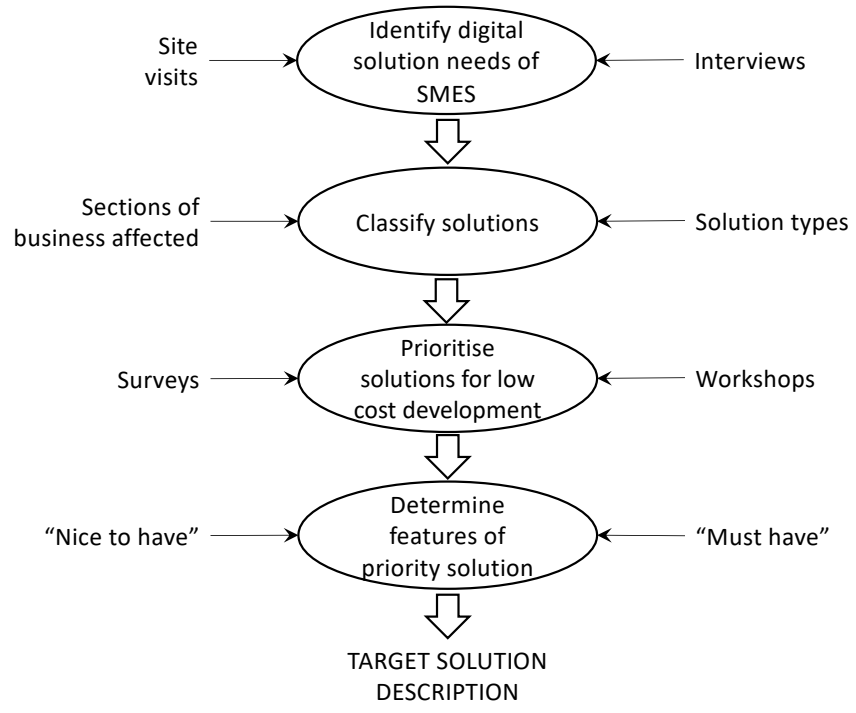

Fig. 2 Method to prioritise required digital manufacturing solutions for SMEs [13]

The method as shown in Figure 2 consists of four stages:

1. Identification of digital manufacturing solution needs of SMEs (described in section 3.2)

2. Classification of the identified solutions (described in section 3.3)

3. Prioritisation of solutions for low cost development (described in section 3.4)

4. Determination of specifications of high priority solutions (left for future work as described in section 5.2)

\subsection{Identification of Potential Digital Manufacturing Solutions}

An initial set of digital manufacturing solutions was identified by the analysis of a historic data set generated by the consulting activities of IfM Education and Consultancy Services (IfM ECS), a consultancy linked to the University of Cambridge. Over 500 cases of so called one day interventions with manufacturing SMEs from 
the UK were evaluated. During these consultations, the companies identify problem areas in their operations and plan solutions. While this is not strictly focussed on digitalisation, many cases in the past led to high-tech solutions, which were added to the initial catalogue.

This catalogue is validated in research workshops with a manufacturing SME audience. At the beginning of each workshop, the participants answer the question "What current challenge in your business would you like to address with a digital solution this year?" as free text input. The answers are compared with the existing items in the catalogue. If the proposed activity is new and not implied by an existing item, it is added. The set is updated with each research cycle and is expected to converge.

\subsection{Classification of Digital Manufacturing Solutions}

The large number and variety of digital solutions in the catalogue evokes the need for a filtering procedure, so that the workshop participants do not need to evaluate every item in the short duration of the workshop. One way of filtering is to only consider solutions that are aligned with the individual company's business model. During the tool assessment described in section 3.1 we identified that the IfM ECS "Prioritisation Tool" provides a framework that allows to do this. Using their approach, we classify the items in the catalogue by their potential to improve a company's "Order Winners" (OWs) or reduce their "Business Constraints" (BCs). OWs are factors that drive a company's order volume whereas BCs are factors that hinder a company's growth $[7,8]$. The categories are further divided into 5 subcategories each (see table $3)$.

Table 3 Categories for the classification of the items in the solutions catalogue

\begin{tabular}{|l|l|}
\hline Order Winners (OWs) & Business Constraints (BCs) \\
\hline \hline Price & People and Information \\
\hline Quality & Plant and Equipment \\
\hline Unique Value & Supply Chain \\
\hline Delivery & Demand \\
\hline Ethical Position & Cash \\
\hline
\end{tabular}

The initial categorisation of the solutions was done by two experienced consultants and a researcher independently. It was guided by detailed descriptions for each of the subcategories. The underlying question was "could this solution help improving the $\mathrm{OW} /$ reducing the $\mathrm{BC}$ ?". The independent results were then discussed and a consensus reached for the categorisation of each item in the catalogue. An item can be connected to multiple OWs and BCs. This is expected, because digital manufacturing projects rarely only improve one isolated process. 


\subsection{Company Priorities}

To guide the research into low cost digital manufacturing solutions for SMEs, one objective of our requirements study is to identify solutions that are commonly demanded by companies. During the workshop, these priorities are captured through a dot-sticking exercise. In a first step every participant chooses the top OW and BC for their company from experience. Then they consider the digital manufacturing solutions that are linked to the respective OW and BC. The participants select up to three solutions that they feel are most urgent (cue: "We really need this") and mark these by sticking a golden star next to them. In order to capture lower priorities as well (cue: "This sounds useful, but it is not a priority"), the participants also select up to 6 more solutions which they mark by sticking a blue dot next to them. This procedure is repeated for both the selected OW and BC.

The data is evaluated by ranking the solutions by the number of stars they received as well as by the cumulated number of markers (stars and dots combined). This allows to identify both the highest priority solutions and solutions that would benefit a very broad group of companies (but are not ranked as highly individually).

\section{Results \& Discussion}

This section shows the first results of the prioritisation exercise described in section 3.4. At this point, the catalogue comprises 51 items. Three research workshops with a total of 40 SME participants were conducted so far. More workshops to update the reference set are currently being run and it also gets updated with each research activity. The most up to date list can be accessed at https://www.digitalshoestring.net.

Figure 4 shows the results of the top 10 prioritised digital manufacturing solutions sorted by which solutions received the most "star" markings (dark grey bar), where participants felt that this solution would give them a huge benefit. Figure 4 shows the top 10 by the combined score of high priority (dark grey bar) and "interesting" (light grey bar) markings.

There is a large overlap between the two rankings. This indicates that the high priority solutions are relevant to a large group of participants. In both cases, the top 4 items are the same, indicating very high priority. Seven items appear in both sets, and three are unique to each of the sets, leading to a total "priority set" of thirteen items. The items can be further grouped by their intrusiveness to existing operations. Data capture and information display do not disturb the current workflow. Data analysis and decision algorithms can influence how human operators act, and thus have an indirect influence on operations. Digital manufacturing solutions that control assets can have a direct influence on operations without a human element.

Below are the 13 identified priority solutions sorted into these groups. 


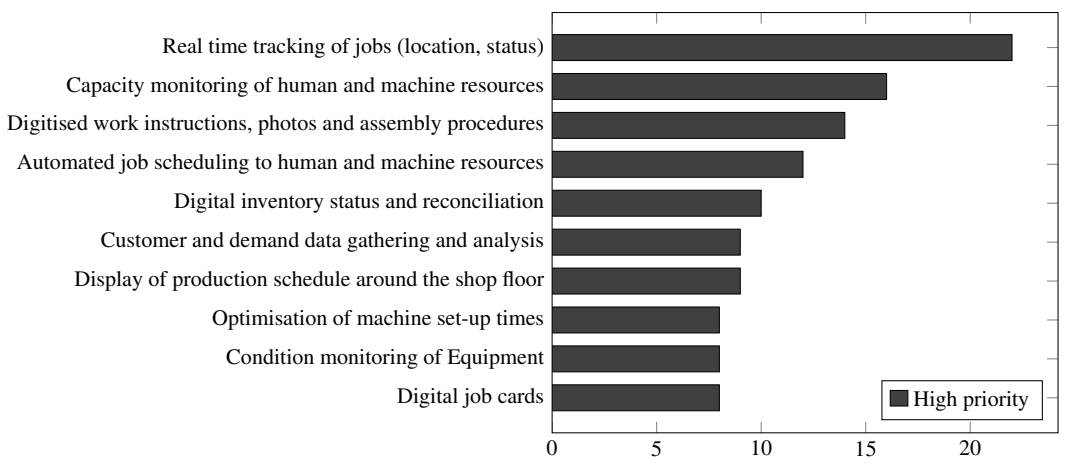

Fig. 3 The top 10 sorted by only the high priority indication

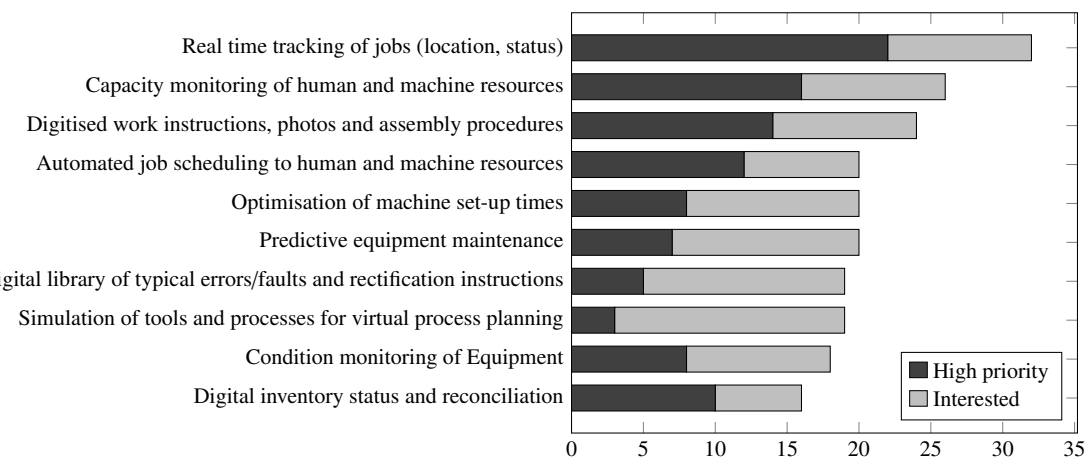

Fig. 4 The top 10 sorted by high priority and interest combined

Data capture and information display

- Real time tracking of jobs (location, status)

- Capacity monitoring of human and machine resources

- Digitised work instructions, photos and assembly procedures

- Display of production schedule around the shop floor

- Condition monitoring of Equipment

- Digital job cards

- Digital library of typical errors/faults and rectification instructions

Data analysis and decisions

- Automated job scheduling to human and machine resources

- Digital inventory status and reconciliation

- Customer and demand data gathering and analysis

- Optimisation of machine set-up times

- Predictive equipment maintenance

- Simulation of tools and processes for virtual process planning

None of the solutions associated with direct asset control were ranked in the top 13 by the participants. 
The data shows that the majority of participants prefer options that have a low risk of disturbing the current operations. This is not surprising: SMEs rarely build new production lines from scratch and any disturbance to existing operations can be a threat to the company. The highest ranked solution areas focus on display or analysis of data; the control remains with a human operator or the established control system. This could be indicative of a general distrust in digital technologies. However, low cost and low risk data capture and display solutions could provide a learning environment for the companies to gain trust in the technologies and to build the skills necessary to initiate larger digital manufacturing activities later on.

\section{Conclusion}

\subsection{Making Use of the Results}

The results of the requirements analysis will be used to guide further developments in the Digital Manufacturing on a Shoestring project. They can also be used more broadly to identify interesting and relevant research opportunities in the area of digital manufacturing technologies.

The feedback received by participants in the research workshops shows that many SMEs have little overview of their options regarding digital manufacturing. The results of this study can help decision makers to investigate potential activities within their firm by checking if they have similar needs to the majority companies. This could also lead to new industrial partnerships being formed to co-develop solutions where an individual firm does not have sufficient resources.

\subsection{Next Steps}

At the time of writing, the data capture is focussed on local industry groups across the UK. The next steps for the application of this research tool are to raise the granularity of the collected data by also capturing the industry sectors that the participants are active in. Furthermore, we will deploy the workshop to other economic areas such as India and Australia to look for similarities between emerging and established market companies.

Our initial catalogue of standardised digital manufacturing solutions is only the first step. We plan to develop a refined system to classify the projects based on their inherent characteristics, enabling companies to choose their projects with higher accuracy. In addition, each solution in the catalogue will include technical specifications that are needed to make it feasible to SMEs.

The other work streams of the Digital Manufacturing on a Shoestring project will use the outcome of this study to develop an architecture for the gradual technical 
implementation of digital manufacturing solutions in SMEs and to identify ways to implement the digital manufacturing solutions from the catalogue in pilot projects with manufacturing SMEs.

\section{References}

[1] Bi, Z., Liu, Y., Baumgartner, B., Culver, E., Sorokin, J., Peters, A., Cox, B., Hunnicutt, J., Yurek, J., O'Shaughnessey, S.: Reusing industrial robots to achieve sustainability in small and medium-sized enterprises (SMEs). Industrial Robot: An International Journal 42(3), 264-273 (2015). DOI 10.1108/IR12-2014-0441. URL http://www.emeraldinsight.com/doi/10.1108/IR-122014-0441

[2] Brunoe, T.D., Nielsen, K.: Complexity Management in Mass Customization SMEs. Procedia CIRP 51, 38-43 (2016). DOI 10.1016/j.procir.2016.05.099

[3] CBI: From Ostrich to Magpie - INCREASING BUSINESS TAKE-UP OF PROVEN IDEAS AND TECHNOLOGIES. Tech. Rep. November (2017). URL https://www.cbi.org.uk/articles/from-ostrich-to-magpieincreasing-business-take-up-of-proven-ideas-and-technologies/

[4] Ciliberti, F., Pontrandolfo, P., Scozzi, B.: Investigating corporate social responsibility in supply chains: a SME perspective. Journal of Cleaner Production 16(15), 1579-1588 (2008). DOI 10.1016/j.jclepro.2008.04.016

[5] Digitalshoestring.net: Digital Manufacturing on a Shoestring (2019). URL https://www.digitalshoestring.net

[6] EPSRC: EPSRC Details of Grant [Digital Shoestring] (2018). URL https://gow.epsrc.ukri.org/NGBOViewGrant.aspx?GrantRef=EP/R032777/1

[7] Ford, D., Caldwell, P., Stevens, T.: A new diagnostic and action planning tool for Small and Medium-sized manufacturers. In: 3rd International Conference for Manufacturing Research, ICMR 2005. Cranfield (2005)

[8] Ford, D., Caldwell, P., Stevens, T.: In-Site: the development of a new diagnostic and action planning tool for small and medium-sized manufacturers. In: 7th International Conference on Stimulating Manufacturing Excellence in Small and Medium Enterprises, pp. 252-259. Glasgow (2005)

[9] Goerzig, D., Lucke, D., Lenz, J., Denner, T., Lickefett, M., Bauernhansl, T.: Engineering environment for production system planning in small and medium enterprises. Procedia CIRP 33, 111-114 (2015). DOI 10.1016/j.procir.2015.06.021. URL http://dx.doi.org/10.1016/j.procir.2015.06.021

[10] Grube Hansen, D., Malik, A.A., Bilberg, A.: Generic Challenges and Automation Solutions in Manufacturing SMEs. In: 28TH DAAAM INTERNATIONAL SYMPOSIUM ON INTELLIGENT MANUFACTURING AND AUTOMATION, pp. 11611169 (2017). DOI 10.2507/28th.daaam.proceedings.161. URL http://www.daaam.info/Downloads/Pdfs/proceedings/proceedings_2017/161.pdf 
[11] House of Commons: Small businesses and productivity Fifteenth Report of Session 2017-19 HC 807. Tech. rep. (2018). URL https://publications.parliament.uk/pa/cm201719/cmselect/cmbeis/807/807.pdf

[12] Matt, D.T., Rauch, E.: Implementation of lean production in small sized enterprises. Procedia CIRP 12, 420-425 (2013). DOI 10.1016/j.procir.2013.09.072. URL http://dx.doi.org/10.1016/j.procir.2013.09.072

[13] McFarlane, D., Ratchev, S., Thorne, A., Parlikad, A., de Silva, L., Schönfuß, B., Hawkridge, G., Terrazas, G., Tlegenov, Y.: Digital Manufacturing on a Shoestring: Low Cost Digital Solutions for SMEs. In: Submitted to SOHOMA 2019

[14] Publications Office of the European Union: User Guide to the SME Definition (2015). URL http://ec.europa.eu/regional_policy/sources/conferences/stateaid/sme/smedefinitionguide_en.pdf

[15] Rhodes, C.: Business statistics - HoC briefing paper, Dec 2018. Tech. Rep. 1 (2018). DOI 10.1111/j.1467-9922.2009.00554.x. URL http://researchbriefings.files.parliament.uk/documents/SN06152/SN06152.pdf

[16] Saam, M., Viete, S., Schiel, S.: Digitalisierung im Mittelstand: Status Quo, aktuelle Entwicklungen und Herausforderungen: Forschungsprojekt im Auftrag der KfW Bankengruppe. Tech. rep. (2016). URL https://www.kfw.de/PDF/Download-Center/Konzernthemen/Research/PDFDokumente-Studien-und-Materialien/Digitalisierung-im-Mittelstand.pdf 